Document downloaded from:

http://hdl.handle.net/10251/65700

This paper must be cited as:

Martínez Millana, A.; Fico, G.; Fernández Llatas, C.; Traver Salcedo, V. (2015).

Performance assessment of a closed-loop system for diabetes management. Medical and Biological Engineering and Computing. 53(12):1295-1303. doi:10.1007/s11517-015-1245-3.

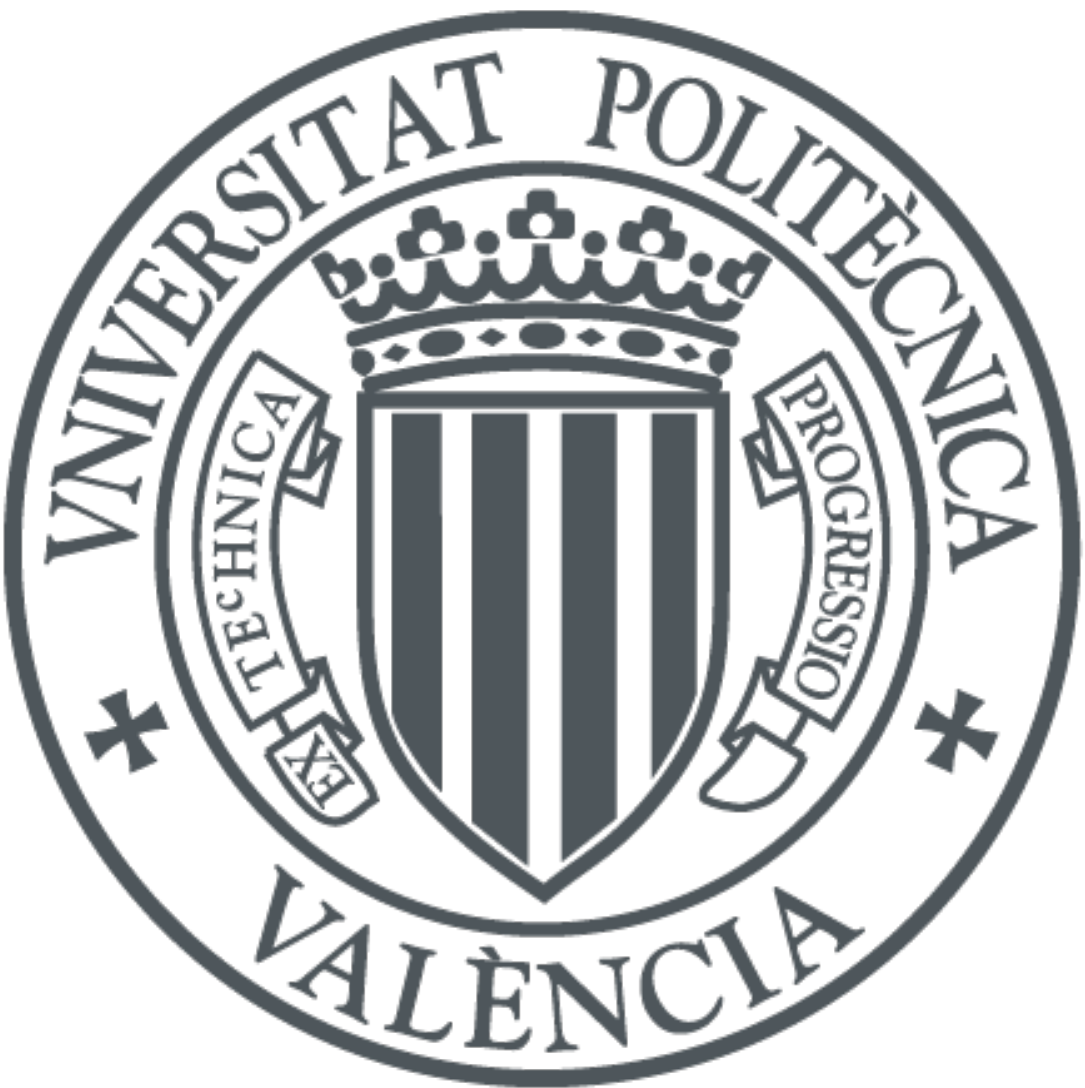

The final publication is available at

http://dx.doi.org/10.1007/s11517-015-1245-3

Copyright Springer Verlag (Germany)

Additional Information 


\title{
PERFORMANCE ASSESSMENT OF A CLOSED-LOOP SYSTEM FOR DIABETES MANAGEMENT
}

\author{
Martinez-Millana A. ${ }^{1}$, Fico G. ${ }^{2}$, Fernández-Llatas C. PhD, Traver V. PhD, ${ }^{1}$ \\ ${ }^{1}$ ITACA - Health and Wellbeing Technologies, Universidad Politécnica de Valencia, \\ Valencia, Spain \\ 2 Life Supporting Technologies, Universidad Politécnica de Madrid \\ Madrid Spain \\ Contact author: Martinez-Millana A., 0034963877606 \\ Universidad Politécnica de Valencia Camino de Vera s/n 46022 Valencia
}

\section{SUMMARY}

Telemedicine systems can play an important role in the management of diabetes, a chronic condition that is increasing worldwide. Evaluations on the consistency of information across these systems and on their performance in a real situation are still missing. This paper presents a remote monitoring system for diabetes management based on physiological sensors, mobile technologies and patient/doctor applications over a service oriented architecture that has been evaluated in an international trial (83905 operation records). The proposed system integrates three types of running environments and data engines in a single service oriented architecture. This feature is used to assess key performance indicators comparing them with other type of architectures. Data sustainability across the applications has been evaluated showing better outcomes for full integrated sensors. At the same time, runtime performance of clients has been assessed spotting no differences regarding the operative environment.

\section{INTRODUCTION}

Diabetes is defined as a set of syndromes where high blood glucose levels are the common factor. Inefficiency or absence of endogen insulin leads to metabolic disorders which cause micro and macro vascular complications in both short and long term [1]. A good quality of life, together with an adequate adherence to the medical treatment can delay the rise of complications [2].

The management of this disease has improved with the arrival of new monitoring systems, insulin pumps and oral drugs. From the handmade logbook reporting to stem-cells research, a wide variety of medical technologies are applied to maintain patient health. The healthcare industry and academics are fostering improvements in monitoring technologies: recently, the accuracy and reliability of a fibercoupled optical continuous glucose sensor was tested for 14 consecutive days in a clinical trial, showing promising results [3]; closed-loop artificial pancreas [4] can significantly reduce glucose variability, regardless patient adherence to treatment. Nevertheless, the patient should not be taken out of the equation. A recent study evaluated the capability of 25 diabetic patients to adjust the subcutaneous insulin infusion therapy and avoid glycemic excursions, proving that an information management system can improved glycemic control by performing accurate adjustments to the insulin delivery [5].

While the literature is progressively focusing on the cost-effectiveness of applying new technologies to diabetes management [6] [7], the focus should be on promoting patient education and consciousness to deal with the disease. In the case of non-communicable diseases, where long duration and slow progression require concrete actions on patient lifestyle, the Information and Communication Technologies (ICT) may contribute to provide the individual and supporting careers with solutions to manage therapies, increase awareness and promote healthy selfmanagement habits [7] [8].

One of the purposes of remote monitoring in diabetes is to prevent a worsening condition and hospitalization due to long-period hyperglycemias and decompensation by providing patient data to care providers [9], which may also lead to reducing the high economic health costs [6]. Besides educational and telephone aid, patient self-management support may be augmented by using homebased technologies that generate data points which providers can potentially use to make more timely changes in the patients' care. The effectiveness of a secure e-mail attention program in diabetic population has been demonstrated [10], nevertheless authors point out the need for embedding such communication channels within a continuous self-management system.

ICT solutions are presented as key solutions for managing diabetes [11]. First interventions used the Internet and Public Switched Telephone Network in urban and rural environments [12] [13]. Later on, mobile networks based on GPRS [4] [14] [15] and internet portals [16] [17] were used as new paradigms of the application of ICT in diabetes management. More recently 
comparative frameworks [18] have described the benefits of using mobile technologies in the management of chronic conditions and other authors have suggested the implementation of new communication architectures based on services (SOA) [19].

The impact of telemedicine interventions is highly dependent on the architecture and performance of the system. The variety of results achieved in the literature [20] could be better understood by contextualizing each intervention in terms of system implementation and performance. There are qualitative and quantitative tools to measure the user response to a single tool or system [21], however, the technical implementation and evaluation should be presented to add significance to the results of a telemedicine intervention. The performance should be assessed using specific benchmarks, known as Key Performance Indicators (KPI), that describe the quality of service. Typical examples of KPIs include cost, security, reliability, and delays.

The rationale of this paper is to present a new system for diabetes remote monitoring oriented to patient self-management based on a SOA and to introduce a technical assessment of the system performance in a trial with 30 type 1 and 2 diabetes patients and up to 83905 interaction records. The system uses home and portable devices for monitoring blood glucose, physical activity, weight, blood pressure and applications to record data (food intake, medication, educational level and lifestyle). Unlike the related work, the insulin pump device is discarded as the patient himself is the booster of the treatment (according to doctor's guidelines). The presented SOA is based on internet protocol communication which merges different operative systems and software environments (Windows Mobile, iOS, Apache Tomcat and .NET) and is evaluated using existing KPIs under specific domains [22]. Clinical and usability assessments are out of the scope of this research work. Records of data, synchronization logs and services invocations have been analyzed to evaluate the effectiveness of the proposed solution from a performance and data aggregation perspective.

\section{METHODS}

The architecture of the telemonitoring system consists of three main blocks, shown in Figure 1. In the bottom square: patient devices with mobile and desktop applications to retrieve data from sensors and to self-manage the disease by recording events, reading prescriptions from the doctor side and showing historic trends. Top right the Control Panel (CP), from which healthcare professionals are able to supervise patient evolution, prescribe treatments and modify therapies. And top left the system central server where all the communicating functionalities and intelligent modules are hosted to provide a user-blinded closed-loop solution to all users: both patients and care providers.

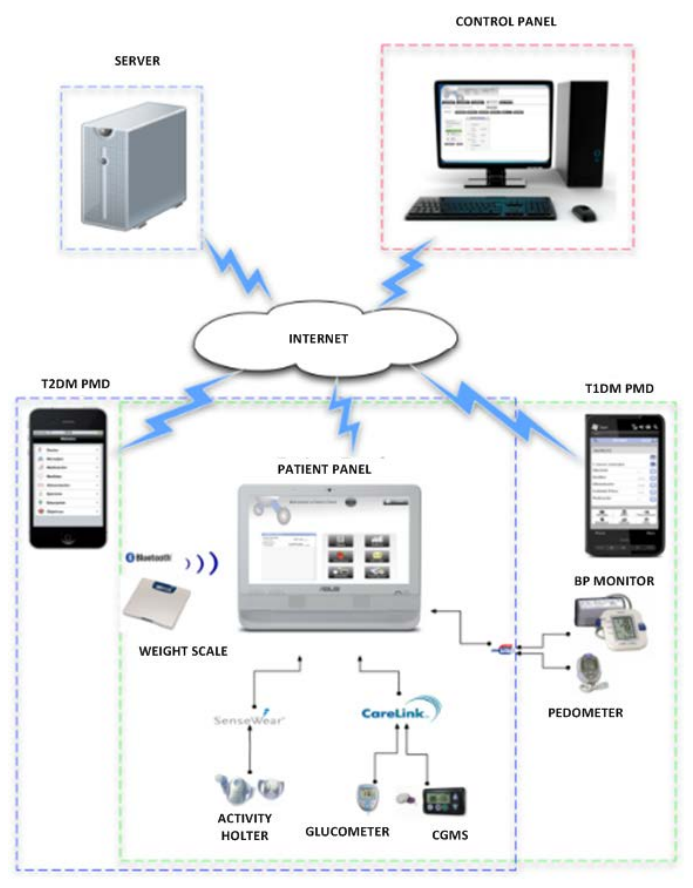

Figure 1. System schema combining the components devoted for patients, doctors and server. Sensors are connected to the Patient Panel through the interfaces depicted in Section 2.2. All the communications are held by the Internet Protocol.

Single features for each type of user were translated into requirements and then deployed as tools to run over multiple platforms (Figure 1). This process was made under User Centered Design (UCD) techniques [23]. These tools were developed within Application Program Interfaces (APIs) and scaled to provide multiple levels of interactions, depending on the type of diabetes and the type of user. The information generated was sent via web services to healthcare professionals involved in the follow up process. Two Patient Mobile Devices (PMD) contained native applications to record and display prescriptions fixed by the doctor in the $\mathrm{CP}$, trends from sensor and lifestyle variables (food intake and medication) and educational material. T1DM patients were provided with a HTC (WM 6.1) and T2DM an iPhone 3G (iOS 4.2.1). Patient Panel (PP) was a table/desktop application that both type of users were able to use to download sensor data (sensor platform is further detailed in point 2.4) from each device and upload it to the system server so it was 
accessible to both the physicians application and the patient mobile application.

\subsection{Service Oriented Architecture}

The main technical challenge consisted in storing this kind of information in a common Personal Health Record (PHR), enabling different interaction from all the modules within the system. Client applications (patient and doctor) are connected through a distributed SOA which contains a central data base and surrounding modules to build up a secure and scalable platform of functionalities for data analysis and exchange.

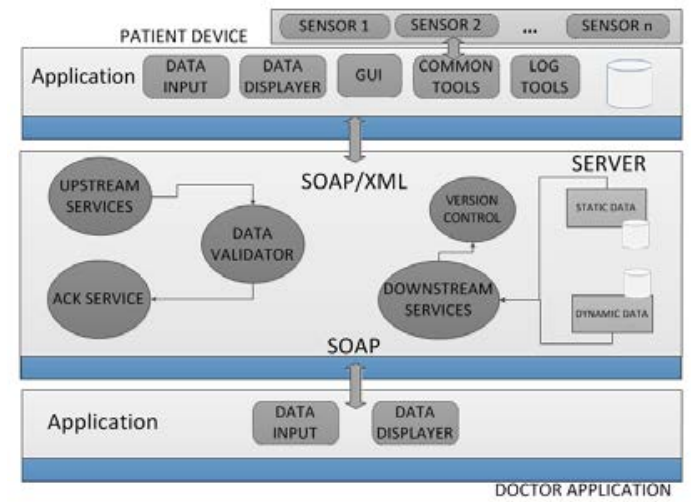

Figure 2. System architecture is built up by three layers connecting different technologies through a web service implementation.

Up to seven modules were in charge of administrating the central data base, performing data analysis, controlling patient compliance and treatment follow up awareness in terms of the uploaded data. This approach was designed to provide consistent security features (access and integrity) at the same time to all data entities.

To meet system requirements in the architecture drawn in Figure 2, web services were developed to be invoked from different technologies through a combination of SOAP/XML and WSLD protocols. A total of forty-five methods were designed, implemented and tested. Information was embedded into XML files and sent as serialized or compact objects depending on the connection speed rate.

Different client applications (iOS, .NET, java) are integrated in this platform using several data engines (SQLite, SQLCE, MySQL). Despite the similar data type definitions for these technologies, many troubles were found in the integration of some data-types (mostly related to the number of bytes contained Integer and Float values). Thus, all services were developed to work with the common data type across mentioned platform (32 byte string), and then convert the value locally depending on the type of client implementation.

\subsection{Sustainable Data Management and Storage}

Several approaches to support easy information acquisition have been developed to guarantee sustainable data storage. Giving the user the right information at the right moment may enable patients to achieve better results on health indicators.

To maintain data integrity and keep a stable PHR synchronization among components, an ad-hoc acknowledgment protocol based on global unique identifiers was developed. To be able to use communication services, each application had to be registered prior to normal service requests. The registration process consisted in providing the patient with a username and a password to log into the application. If credentials matched, the application was registered into the server and services enabled to start exchanging sensitive information only to that unique patient device. Subsequent access to the application was made possible by inputting a personal identification number (configurable by patients).

Within the mentioned services, a set of three services were developed to deal with sensor data. Sensor synchronization was designed to occur in a wide variety of time intervals. Against plain-text information wrapped into XMLs, sensor data was sorted into a custom format vector to avoid service overloads (headers and identifiers repeated for each measurement). For instance, Continuous Glucose Monitoring System (CGMS) data recorded during two weeks could be uploaded at once containing more than four thousand measurements as well as pedometer data corresponding to one day, containing twentyfour values.

\begin{tabular}{llc}
\hline ID & Sensor & Group \\
\hline 1 & Medtronic GuardianRT $®$ CGMS & 2 \\
2 & Bayer тм Contour-Link Glucometer & 2 \\
3 & SenseWear ArmBand - Physical & 2 \\
& Activity Sensor & \\
4 & OMRON HJ-720IT Pedometer & 1 \\
5 & OMRON MT-10T Blood pressure & 1 \\
6 & AANDD Weight Scale & 3 \\
\hline
\end{tabular}

Table 1. Medical sensors within the platform sorted by the group to which they belong depending on the data access protocol.

Different types of information acquired from several data sources had to be stored in a homogeneous database. ISO 11073 data 
structure standard was used to classify values read from the sensors through an Application Program Interface (API) [27]. The deployed API merged functionalities to hold communications through physical interfaces (Group 1: USB, Group 2: virtual sensors, using proprietary protocol and providing CSV reports, Group 3: Bluetooth) and logical channels, deploying the communication protocols for each sensor. Table 1 shows the correspondence between each sensor and the physical channel implemented.

Automated scripts were implemented to manage the sensor data in a transparent approach for the user. Host application (PP) was deployed to download, store and synchronize sensor data automatically as soon as the patient connected the sensor.

\subsection{Data heterogeneity: management and synchronization.}

The dynamic nature of the medical parameters gathered in the PP and the distributed heterogeneous data from each single sensor and manual record required new approaches to data warehousing and management.

The central data base, contained in the system server, was located in a Windows Server 2008 virtual server in Prage, CZ, separate to hospital networks, due to the multicenter study design and in order to maintain data integrity and avoid integration issues. The data base complied with the security standards on electronic medical data storage (ISO 27001) and privacy of data was ensured through dynamic encryption.

Multi-parametric data coming from devices and sensors (dynamic data) was integrated in a centralized database. The distributed approach enabled dynamic information to be added, modified and displayed at the same time, individually or as a whole in each client application (Figure 2). Therefore, to avoid persistent misleading each value was assigned with a unique identifier allowing them to be distinguished in an efficient and rapid way. Good practices were developed to maximize communication data exchange and memory allocation and an online/offline management system was developed to ensure data synchronization.

During the communication processes between medical devices, data was only transmitted through wireless internet connection (mobile network or WIFI) availability. In case of offline sessions, data was locally stored and sent whenever the connection was available. Only new data (no downloaded or sent previously) was synchronized. Integrity of data and synchronization has been also considered. Furthermore, unique identifiers for patients and parameters were assigned to provide a high integrity of data allowing re-synchronization in case it was needed. Automatic back-up routines were also implemented in the central server to guarantee data loss or corruption recovery.

\subsection{Evaluation}

All screened patients who fulfilled inclusion criteria entered a Baseline Phase of at least 3 days (up to 6 days, based on local monitoring practice). The inclusion criteria for patients was to be categorized for all types of diabetes and to have the ability to use mobile devices and sensors. During the Baseline Phase, patients recorded metabolic data on food and drug intake (through a paper diary), physical activity (through the combined use of OMRON HJ720IT pedometer and Sensewear-Armband), fingerprint glycemic values (Bayer тм Contour Link glucometer), and continuous subcutaneous glucose concentration (Medtronic GuardianRT® Continuous Glucose Monitoring System).

30 patients from three EU countries (Spain, Italy and Czech Republic) were planned to be enrolled for the trial. Up to 33 patients were enrolled finally, from which 3 dropped out, meaning 30 patients completed the trial phase. All patients signed an agreement and were fully informed about the objective of the trial. After the initial baseline, which consisted in a paper track of the monitoring parameters, patients were trained during a two-hour session to use PMD and PP to achieve the recommended goals for four consecutive weeks. Trial duration was $35 \pm 6$ days. All patients were asked to monitor the complete set of metabolic data during the final 3 (6) days of the intervention phase (including the paper diary for food and drug intake).

Four follow-up visits (V0-V3) were established for all subjects. The number of days between the chosen visits depended clinical practice in each country, V1-V0 was $6 \pm 2$, V2-V1 was $20 \pm 4$ and V3-V2 was $6 \pm 2$.

During trials, PMDs, PPs and services usage were evaluated trough the follow up of data increase, services consumption, exceptions rise and memory load (virtual and physical). Daemon services were enabled to record locally and remotely the interaction flows within modules and communications. To perform an automatized analysis, the information was 
recorded in txt files following a structured format.

The ethical committee of each involved hospital approved the study protocol. No ethical issues were found prior and after the realization of the study.

\section{RESULTS}

Prior to trial evaluation, the system was tested in laboratory facilities with real devices, servers and switching 3G/Wi-Fi connections obtaining good results on time delays and performance for a set of benchmarked operations designated as critical. After these tests, the platform was evaluated in an international multi-center openlabel randomized study consisting of two groups, one intervention and other control. Table 2 shows a description of the group which completed the trial. System usefulness and innovative features as data aggregation, PHR tailoring and comprehensive view on health data has been evaluated through a transparent analysis of the components interaction, based on events triggered by patients.

\begin{tabular}{|c|c|c|c|c|c|c|c|}
\hline & \multicolumn{2}{|c|}{ Total $(n=30)$} & \multicolumn{2}{|c|}{$\begin{array}{l}\text { T1DM } \\
(n=20)\end{array}$} & \multicolumn{2}{|c|}{ T2DM-IT $(n=10)$} \\
\hline & & $\mathrm{n}$ & $\%$ & $\mathrm{n}$ & $\%$ & $n$ & $\%$ \\
\hline \multirow{2}{*}{ Gender } & Male & 19 & 63 & 14 & 70 & 10 & 100 \\
\hline & Female & 11 & 37 & 6 & 30 & 0 & 0 \\
\hline \multirow{4}{*}{ Studies } & Undergraduate & 3 & 10 & 3 & 15 & 1 & 10 \\
\hline & Secondary & 10 & 33 & 8 & 40 & 3 & 30 \\
\hline & University & 16 & 53 & 17 & 85 & 4 & 40 \\
\hline & $\mathrm{PhD}$ & 1 & 3 & 1 & 5 & 0 & 0 \\
\hline \multirow{4}{*}{$\begin{array}{c}\text { Marital } \\
\text { Status }\end{array}$} & Single & 14 & 47 & 13 & 65 & 3 & 30 \\
\hline & Married & 13 & 43 & 12 & 60 & 7 & 70 \\
\hline & Divorced & 2 & 7 & 2 & 10 & 0 & 0 \\
\hline & Widowed & 1 & 3 & 0 & 0 & 0 & 0 \\
\hline \multirow{4}{*}{$\begin{array}{l}\text { Commor } \\
\text {-bidities }\end{array}$} & Infraction & 0 & 0 & 0 & 0 & 0 & 0 \\
\hline & Stroke & 2 & 7 & 2 & 10 & 0 & 0 \\
\hline & Renal Failure & 0 & 0 & 0 & 0 & 0 & 0 \\
\hline & Liver Failure & 0 & 0 & 0 & 0 & 0 & 0 \\
\hline \multirow{3}{*}{ Lifestyle } & Smoking & 9 & 30 & 4 & 20 & 5 & 50 \\
\hline & Alcohol & 12 & 40 & 8 & 40 & 4 & 40 \\
\hline & Restrictive Diet & 8 & 27 & 6 & 20 & 2 & 20 \\
\hline
\end{tabular}

Table 2. Study population dataset description in absolute and relative values divided for total population, type 1 diabetes patients (T1DM) and type 2 diabetes patients (T2DM).

The system was used during $35 \pm 6$ days between February 2012 and September 2012 by a total set of 30 patients aged $45 \pm 11$. Table 2 depicts the data set description on gender distribution, studies level, marital status, comorbidities and lifestyle. All the patients were able to perform measurements with the sensors and use mobile and desktop applications, $90 \%$ of the study population had secondary or higher education. Patients were assisted by technical staff and their medical practitioner during the study duration.

The main purpose for the system administration, maintenance and performance was measured and evaluated under a set of Key Performance Indicators (KPI). A KPI is introduced as significant assessing criteria for system performance under a specific domain [22] [24] that may be referred by an absolute or relative value. For the purpose of this evaluation the following KPIs were addressed: total measurements and single entries for data aggregation; computational load, time delay and success rate for system applications performance.

The assessment consisted in the analysis of service invocation logs, application logs and data base mining to evaluate the following two features:

\subsection{Data aggregation from multiple sensors to support health decisions.}

This analysis evaluates platform capability of aggregating data from different sensors, displaying them and making them available to health professionals preserving data integrity. Data has been analyzed for each patient in two levels; in the client application and the server core. Table 3 shows the number of patients which used each device. Through the PP application data was downloaded and aggregated in a local data base (row 3) and then synchronized to the server a total of 124875 measurements. Physicians had straight access to server data through the $\mathrm{CP}$, so that was the total percentage of data available to him (row 3 shows the percentage of the patient data visible to health professionals). Outliers have been removed prior to data statistical analysis, meaning that only patients who used sensors regularly have been included. The group $(n=30)$ was told to use the system to retrieve data from the different sensors (glucose, continuous monitor glucose, physical activity).

\begin{tabular}{|l|c|c|c|c|}
\hline \multicolumn{1}{|c|}{ SENSOR ID } & 1 & 2 & 3 & 4 \\
\hline $\begin{array}{l}\text { Number of } \\
\text { Patients }\end{array}$ & $\begin{array}{c}18 \\
(69 \%)\end{array}$ & $\begin{array}{c}19 \\
(73 \%)\end{array}$ & $\begin{array}{c}12 \\
(46 \%)\end{array}$ & $\begin{array}{c}23 \\
(88 \%)\end{array}$ \\
\hline $\begin{array}{l}\text { Total } \\
\text { Measurements in } \\
\text { Patient Device }\end{array}$ & $\begin{array}{c}4106,3 \pm \\
3647,2\end{array}$ & $\begin{array}{c}94,1 \pm \\
74,1\end{array}$ & $\begin{array}{c}4027 \\
\pm 395\end{array}$ & $33 \pm 7,7$ \\
\hline $\begin{array}{l}\text { Single entries in } \\
\text { Patient Device } \\
\text { (\%) }\end{array}$ & $\begin{array}{c}85,9 \pm \\
32,0\end{array}$ & $\begin{array}{c}83,6 \pm \\
61\end{array}$ & $\begin{array}{c}43,5 \pm \\
5,8\end{array}$ & $\begin{array}{c}95 \pm \\
22,36\end{array}$ \\
\hline $\begin{array}{l}\text { Measurements in } \\
\text { Server (\%) }\end{array}$ & $\begin{array}{c}79,6 \pm \\
27,7\end{array}$ & $\begin{array}{c}87,6 \pm \\
16,3\end{array}$ & $\begin{array}{c}18,4 \pm \\
7,4\end{array}$ & $\begin{array}{c}93,7 \pm \\
14,9\end{array}$ \\
\hline
\end{tabular}

Table 3. Data integrity evaluation in terms on mean and standard deviation for the whole trial evaluation. Sensor ID refers to sensors in Table 1.

Two main families of sensors were defined, F1 containing sensors \#1,\#2 and \#3 for T1DM 
patients and F2 containing \#2, and \#4 for T2DM.

One limitation of the study is that not all the patients used the given sensors, as the second row states. The third row shows the mean and standard deviation of total measurements for each sensor and patient device. As expected, continuous monitors had large quantities of data. The variations found in sensor number 2 are due to the fact that T1DM patients only used it to calibrate sensor number 1 . Forth row shows the percentage of duplicated data (mean $\pm \mathrm{sd}$ ) and the fifth row the percentage of original data that was finally transmitted to the server, and thus was available to the doctor.

\subsection{Uniform access to operational tools.}

Services wrapping complex tasks in an automatic and user-transparent way (e.g.: sensor data synchronization) were been evaluated and analyzed. This evaluation was tackled from two approaches. The first focused on the access to remote functionalities such as web services invocation success, and the second on the performance of the applications in terms of memory (computational) load and time delay on loading the modules in which the applications consisted of.

\begin{tabular}{|l|c|c|c|c|}
\hline & CP & T1DM & T2DM & PP \\
\hline $\begin{array}{l}\text { Computational } \\
\text { load (kB) }\end{array}$ & $\begin{array}{c}120000 \\
(2.9 \%)\end{array}$ & $\begin{array}{c}30000 \\
(7 \%)\end{array}$ & $\begin{array}{c}15000 \\
(3 \%)\end{array}$ & $\begin{array}{c}110000 \\
(2.8 \%)\end{array}$ \\
\hline Time delay (s) & 5,83 & 1,63 & 2,93 & 2,6 \\
\hline
\end{tabular}

Table 4. Application performance parameters of user applications: memory footprint and time delay.

During the study, 83905 calls to web services were performed, from which 63\% $(n=53004)$ were performed by T1DM and 37\% ( $n=30901)$ were performed by T2DM patients. Global service invocations distribution was $3356 \pm 2426$, from which $4077 \pm 2853$ were made by T1DM and $2575 \pm 1639$ were done by T2DM.

From the total of calls, a $97 \%(n=81294)$ were identified as 3G or $\mathrm{WiFi}$ connection, 64\% $(n=51814)$ of them were performed by T1DM and $36 \%(n=29480)$ were made by T2DM. Analysis on the type of connection showed that $69 \%(n=56064)$ of the total of calls were carried out through WiFi connections and 31\% $(n=25230)$ by 3G. Drilling down on the type of connection and type of diabetes, for T1DM patients $71 \%(n=36999)$ were via Wifi and $29 \%$ $(n=14815)$ via 3G. In T2DM, 65\% $(n=19065)$ used Wifi and 35\% ( $n=10415) 3 G$ connection, showing no significant differences.

Regarding the response time, on average, the request delay per call was 1059 ms. For the
iPhone it took $641 \mathrm{~ms}$, and for the HTC was $1303 \mathrm{~ms}$, the largest requests being the ones related to sensor data synchronization.

To value the reported results, the comparative Table 5 shows the most important features of the referenced studies, SMARTDIAB [17], PFTH [26] and METABO, the system assessed in this manuscript. First of all it is clear that there is a lack of parameters in [17] on the technical assessment and report of KPI performance. Second, a better behaviour is found in [26] with respect to the data management, even the amount of the dataset is not comparable (4002 single entries Vs 124875). The unique KPI depicted in the three studies is the system availability, in which the SOA approach shows the best performance rate.

\begin{tabular}{|l|c|c|c|}
\hline \multicolumn{1}{|c|}{$\begin{array}{c}\text { KPI } \begin{array}{c}\text { Intervention } \\
\text { DIAB }\end{array} \\
\begin{array}{l}\text { Number of } \\
\text { patients }\end{array}\end{array}$} & $\begin{array}{c}\text { SMART } \\
\text { PFTH }\end{array}$ & METABO \\
\hline Timespan (days) & - & 24.3 & 30 \\
\hline $\begin{array}{l}\text { Total } \\
\text { Measurements }\end{array}$ & - & 4002 & 124875 \\
\hline $\begin{array}{l}\text { Measurements } \\
\text { per Patient }\end{array}$ & - & $133 \pm 37$ & Table 3 \\
\hline Duplicated Data & - & - & $22,3 \%$ \\
\hline Data Lost & - & $0 \%$ & $30,5 \%$ \\
\hline $\begin{array}{l}\text { Maximum Time } \\
\text { Delay (s) }\end{array}$ & 93.33 & - & 2 \\
\hline $\begin{array}{l}\text { System } \\
\text { Availability }\end{array}$ & $95 \%$ & $96,6 \%$ & $98 \%$ \\
\hline $\begin{array}{l}\text { Computational } \\
\text { Load of the } \\
\text { Applications }\end{array}$ & - & - & Table 4 \\
\hline
\end{tabular}

Table 5. Comparison of the study features and reported KPIs. The kindness of the designed system over the referenced architectures is achieved by improving some of the KPI analyzed Time Delay, System Availability. Other aspects related to data integrity do not achieve the rates reported by [26].

Due to the system specifications is no possible to compare some of the KPI straightforward. For instance, it is senseless to perform a mean of the Measurements per Patient and compare the result with the value from PFTH. However, it can be compared to each sensor, showing that the diabetes system gathers around forty times the data of each patient for sensor 1 .

\section{DISCUSSION}

Telemonitoring solutions for chronic conditions management are being assessed currently through multiple clinical [3][10] and economic studies[6][7]. Some of the preliminary results conclude that the use of these technologies is not related to an improvement in health indicators [20]. Nonetheless, other authors [25] point out that the use of remote monitoring systems has to be selective, prioritizing it for 
empowered patients or who are undergoing in a treatment modification.

The self-management of chronic diseases such as diabetes can be boosted by applying userfriendly systems with a low error rate in communications and excellent performance. Moreover, these technologies would provide large amounts of temporary information to detect patient changes and worsening periods.

In this paper a novel system for diabetes monitoring and management has been presented. The system has introduced two new concepts: the integration of different software environments (enabling patient specific interfaces to be built) through a service oriented architecture based and the creation of a platform of sensors that allows for the sharing of data, making it available for patients and professionals.

In this system, patients have been equipped with mobile devices (T1DM: HTC; T2DM: iPhone 3G), desktop applications and sensors to follow up their medical parameters and normal daily lifestyle. A set of four sensors (Medtronic GuardianRT, Bayer ConoturLink, Sensewear Armband and Omron HJ720 pedometer) has been integrated into the system through the development of a communication API.

Furthermore, the system has been evaluated in a multicenter trial $(n=30)$ during $35 \pm 6$ days within a seven month period. The population sample was categorized by gender, marital status, academic level, presence of comorbidities and lifestyle. No analysis within these groups has been performed during this study.

The evaluation strategy consisted on performing a technical evaluation of the logs registered during trials and data bases. The data aggregation from different sensors has been evaluated analyzing the integrity of data across the platform. Despite the low performance of sensor \#3 (Table 1), a high percentage of nonreplied data from different sensors was found on both patient and doctor applications, pointing out the success on data aggregation.

The integrity of these values on their exchange through the system has been assessed by comparing the number of entries for each patient and sensor. Results show that the vast majority of these values were correctly transmitted and therefore, contribute to highlight the quality of data in terms of integrity and reliability. The best performance has been achieved by sensor \#4, revealing that a better level of integration (Group1 in Table 1) guarantees better level of data aggregation and integrity. The strategy of parsing values to string type and wrap them in ad-hoc vector packets stands as a good strategy to avoid data mismatches. Despite being from Group 2 (Table1), sensor \#3 resulted in an extremely low percentage of successful transmissions to the server (Table 3). The reason could be that in the high number of duplicated entries caused by this type of sensor, and errors in the particular logic of the upstream methods that should be revised after the evaluation (the API share methods within the same sensor group). In comparison to the results achieved by [26], the system shows a bad performance in Data KPIs, leading to conclude that more methods to guarantee a correct data acquisition should be implemented. Since the availability of the services and their successful transactions rate achieves a $98 \%$, the problems underneath the incomplete data transfer are localized in the communication protocols with the sensors at a device level. Therefore, better integrity and reliability features (CRC checksum, logical channel control) should be addressed.

Subsequently, server and application logs have been analyzed to evaluate uniform access to technical components to hide operational complexity. Applications had a good performance on each operative system and device, as memory footprint and delays between modules parameters reveal (Table 4). However, problems were found on T1DM application (running on Windows Mobile 6.5) as the duration of the session was increasing, thus, authors conclude that this operative system is not suitable for complex graphical resources management and XML exchange through web services. Communications used within the trial via web services were handled correctly with a $98 \%$ of successful transactions, even via $3 G$ or Wifi connections from all the system components. The average time delay is acceptable ( $<2$ s). Therefore masking complex operations with web services in a SOA system has been demonstrated to have a good performance with respect to other architectures [17] [26], as Table 5 shows.

The study does not refer to usability and user satisfaction metrics, which could enhance the quality of the result by categorizing the system performance towards system acceptance. The main limitation of the study is found in the sensor assignation for each type of user. Due to logistic issues, some sensors had to be replaced between the initial sets (F1 and F2) and therefore it has not been possible to report comparisons between these two groups. Another limitation is found on the way to measure 
security and reliability of the system. Other mechanisms should be added to assess in-depth the integrity of data, such as quality vector methodologies. The next step towards a full evaluation is to include a user satisfaction evaluation and clinical assessment of the proposed solution.

\section{CONCLUSION}

This paper has demonstrated that web services are suitable to build complex systems which integrate desktop and mobile devices and physiological sensors. Literature is currently focused on determining the cost-effectiveness of tele-monitoring systems without approaching a technical evaluation, as it done is this paper. It also presents a novel SOA that merges different types of technologies and monitoring sensors. This paper will help other researchers to perform similar evaluations, focusing on the same KPI, and also to enhance techniques and methods to evaluate the performance of remote monitoring systems in order to set a quantitative framework for assessing the performance of remote monitoring systems. Future work claims to perform a clinical evaluation of the proposed system in a large study. This study should evaluate the impact of this system in health indicators such HbA1C, hospitalizations, body mass gain and QALY. Another field for future work will be adding qualitative results on user satisfaction and usefulness of the system.

\section{ACKNOWLEDGMENTS}

The authors wish to acknowledge the consortium of the METABO project (funded by the European Commission, Grant nr. 216270) for their commitment during concept development and trial execution.

\section{REFERENCES}

[1] Harrison's Principles of Internal Medicine. McGraw-Hill ISBN 978-0071748896. Ed. July 2011

[2] The Diabetes Control and Complications Trial Research Group. "The effect of intensive treatment of diabetes on the development and progression of longterm complications in insulin-dependent diabetes mellitus”.N Engl J Med. 1993 Sep 30;329(14):977-86 [3] Achim Josef Müller, Monika Knuth, Katharina Sibylle Nikolaus, Roland Krivánek, Frank Küster and Christoph Hasslacher. "First Clinical Evaluation of a New Percutaneous Optical Fiber Glucose Sensor for Continuous Glucose Monitoring in Diabetes " J Diabetes Sci Technol 2013 7: 13

[4] Gómez E.J., Hernando M.E., et altres. "The INCA System: A Further Step Towards a Telemedical Artificial Pancreas". IEEE Trans. Inf. Technol. Biomed vol. 12, no. 4 pp 470-479, Jul 2008

[5] Andreas Reichel, Hannes Rietzsch, Barbara Ludwig, Katrin Röthig, Annette Moritz, and Stefan
R. Bornstein. "Self-Adjustment of Insulin Dose Using Graphically Depicted Self-Monitoring of Blood Glucose Measurements in Patients with Type 1 Diabetes Mellitus” J Diabetes Sci Technol 2013;7(1):156-162

[6] Obstfelder A., Engeseth K.H., Wynn R. Characteristic of succesfully implemented telemedical applications (2007). Implement Sci. 2:25 [7] Ryan et altres. Clinical and cost effectiveness of mobile phone supported self-monitoring of asthma: multicenter randomized controlled trial (2012). BMJ;344:e1756.

[8] D.S.Schade et alters. To pump or not to pump (2005). Diabetes Technology \& Therapeutics. 7:845848.

[9] WakefieldBonnie J. et alters. Effect of Home Telemonitoring on Glycemic and Blood Pressure Control in Primary Care Clinic Patients with Diabetes (2014). Telemedicine and e-Health. 20(3): 199-205. doi:10.1089/tmj.2013.0151.

[10] Zhou YY, Kanter MH, Wang JJ, Garrido T. Improved quality at Kaiser Permanente through email between physicians and patients.

Health Affairs July 2010; 29,7:1370-5.

[11] David C. Klonof "Twelve Modern Digital Technologies That Are Transforming Decision Making for Diabetes and All Areas of Health Care.” J Diabetes Sci Technol 2013;7(2):291-295

[12] Trief P.M, P.C. Morin, Izquierdo R., Teresi J.A., Eimicke J.P., Goland R., Starren J., Shea S. and Winstock R.S. "Depression and glycaemic control in elderly etchnically diverse patients with diabetes: The IDEATel project”. Diabetes Care, vol 29, no 4, pp 830-835,2006

[13] Bellazzi, R., Larizza C., Montani A., et altres. "A telemedicine support dor diabetes management: The T-IDDM Project.” Comput. Methods Programs Biomed, vol 69,pp147-161,2002

[14] Shantau et altres. Using Mobile Health to Support Chronic Care Model: Developing an Institutional Model (2012). International Journal of Telemedicine and Applications, 871925.

[15] García Saez et altres. Architecture of a wireless Personal Assistant for Telemedical diabetes care (2009). International Journal of Medical Informatics 9;78:391-403

[16] Lanzola $G$ et altres. Going mobile with a multiaccess service for the management of diabetic patients (2007). J Diabetes Sci Technol. Sep;1(5):730-7.

[17] Stravroula G., Christos S. Bartsocas et altres. "SMARTDIAB: A communication and Information Technology Approach for the Intelligent Monitoring, Management and Follow-up of Type 1 Diabetes Patients” IEEE Trans. Inf. Technol. Biomed vol. 14, no. 3 pp 622-633, (May 2010)

[18] Pravin P. et altres. A framework for the comparison of mobile patient monitoring systems. Journal of Biomedical Informatics 45 544556(2012).

[19]Ing-Yi Chen et alters. Pervasive Digital Monitoring and Transmission of Pre-Care Patient Biostatics with an OSGi, MOM and SOA Based Remote Health Care System. Proceedings of the Sixth Annual IEEE International Conference on PerCom. Hong Kong; 2008. 
[20] Cartwright et altres. Effect of telehealth on quality of life and psychological outcomes over 12 months: nested study of patient reported outcomes in a pragmatic, cluster randomised controlled trial. BMJ 2013; 346:f653.

[21] van der Weegen et altres. "The development of a mobile monitoring and feedback tool to stimulate physical activity of people with a chronic disease in primary care: a user-centered design”. JMIR. 2013 Jul 2;1(2):e8.

[22] K. Boloor, R. Chirkova, T. Salo, and Y. Viniotis, "Analysis of response time percentile service level agreements in soa-based applications," Global Telecommunications Conference (GLOBECOM 2011), IEEE, dec. 2011, pp. 1 -6

[23] Fico, G., Fioravanti, A., Arredondo Waldmeyer, M. T., Leuteritz, J. P., Guillén, A., Fernandez, D.. A user centered design approach for patient interfaces to a diabetes IT platform. Conf Proc IEEE Eng Med Biol Soc. 2011;1169-72

[24] Xiaowan Ke, Wenjing Li et altres. WCDMA KPI Framework Definition Methods and Applications. ICCET Proceedings, 2010 V4-471 475.

[25] Ma C et altres. Empowering patients with essential information and communication support in the context of diabetes (2006) . Int J Med Inform. Aug;75(8):577-96.

[26]Winkler S. et altres. A new telemonitoring system intended for chronic heart failure patients using mobile technology - Feasibility Study (2011). International Journal of Cardiology 153 55-58

[27] Fioravanti, A., Fico, G., Arredondo, M. T., Salvi, D., \& Villalar, J. L. (2010, August). Integration of heterogeneous biomedical sensors into an ISO/IEEE 11073 compliant application. In Engineering in Medicine and Biology Society (EMBC), 2010 Annual International Conference of the IEEE (pp. 10491052). 